\title{
ALGEBRAIC EQUIVALENCE OF QUASINORMAL OPERATORS
}

\author{
KUNG-YEU CHEN
}

\begin{abstract}
Let $T_{j}=N_{j} \oplus\left(S \otimes A_{j}\right)$ be quasinormal, where $N_{j}$ is normal and $A_{j}$ is a positive definite operator, $j=1,2$. We show that $T_{1}$ is algebraically equivalent to $T_{2}$ if and only if $\sigma\left(A_{1}\right)=\sigma\left(A_{2}\right)$ and $\sigma\left(N_{1}\right) \backslash \sigma_{a p}\left(S \otimes A_{1}\right)=\sigma\left(N_{2}\right) \backslash \sigma_{a p}\left(S \otimes A_{2}\right)$. This generalizes the corresponding result for normal and isometric operators.
\end{abstract}

\section{Introduction}

For any operator $T$, let $C^{*}(T)$ be the $C^{*}$-algebra generated by $T$ and $I$. In other words, $C^{*}(T)$ is the norm-closure of operators of the from $p\left(T, T^{*}\right)$, where $p$ is any polynomial in two noncommuting variables. Two operators $T_{1}$ and $T_{2}$ are algebraically equivalent if there is a *isomorphism between $C^{*}\left(T_{1}\right)$ and $C^{*}\left(T_{2}\right)$ that sends $T_{1}$ to $T_{2}$. It is easy to prove that two normal operators $T_{1}$ and $T_{2}$ are algebraically equivalent if and only if they have the same spectrum (Proposition 2.1). It was shown by $\mathbb{L}$. A. Coburn [3, Theorem 3] that each nonunitary isometry is algebraically equivalent to the simple unilateral shift $S$. The condition on the algebraic equivalence of isometries follows easily (Proposition 2.2). These suggest the following question: when are two quasinormal operators algebrically equivalent? In this paper, we completely solve this problem by using the structure of quasinormal operators (Theorem 2.5).

Before starting our work, we give some notation and terminology. We write $D$ for the open unit disc in the complex plane $\mathbb{C}$ and $\partial D$ for the boundary of $D$. If $H$ is a Hillbert space, we denote by $\mathcal{B}(H)$ the space of all (bounded linear) operators on $H$. If $T$ is an operator on $H, \sigma(T)$ and $\sigma_{a p}(T)$ denote its spectrum and approximate point spectrum. If $H$ is infinite-dimensional, let $\pi(T)$ denote the imageof $T$ in the Calkin algebra $B(H) / \mathcal{K}(H)$ under the natural quotient map $\pi$, where $\mathcal{K}(H)$ is the ideal of compact operators in $\mathcal{B}(H)$. A bounded linear operator $T$ on a complex separable Hilbert space is normal if $T$ and $T^{*}$ commute; $T$ is quasinormal if $T$ and $T^{*} T$ commute. The

Received February 18, 1993; revised March 30, 1993.

1987 Mathematics subject classifications.

Primary 47B99; Secondary 47C05.

Key words and Phrases: quasinormal operators, algebraically equivalent. 
structure of normal operators has been known for a long time. They are multiplication operators on $L^{2}(\mu)$ for some positive measure $\mu$. Quasinormal operators were first studied by $A$. Brown [1] in 1953. Among other things, he obtained a structure theorem for such operators: $T$ is quasinormal if and only if it is unitarily equivalent to an operator of the form $N \oplus(S \otimes A)$, where $N$ is normal and $A$ is a positive definite operator (denoted $A>0)$, that is, $(A x, x)>0$ for any nonzero vector $x$ in the domain of $A$, and

$$
S \otimes A=\left[\begin{array}{ccccccc}
0 & & & & & & \\
A & 0 & & & & & \\
0 & A & 0 & & & & \\
0 & 0 & A & 0 & & \\
& & & . & . & & \\
& & & & . & . & \\
& & & & & . & .
\end{array}\right]
$$

Moreover, in such a decomposition, $N$ and $A$ are uniquely determined by $T$. $N$ and $S \otimes A$ are called the normal and pure parts of $T$, respectively $(S \otimes A$ is pure means that it has no nontrivial reducing subspace on which it is normal).

Finally, we remark that contents of this paper has formed part of the author's $\mathrm{Ph}$. D. thesis [2].

\section{Main Results}

We begin with the following proposition. It is a consequence of the Gelfand theory.

Proposition 2.1. If $N_{1}$ and $N_{2}$ are two normal operators, then $N_{1}$ and $N_{2}$ are algebraically equivalent if and only if $\sigma\left(N_{1}\right)=\sigma\left(N_{2}\right)$.

Proof. One direction is trivial: if $\alpha$ is a *-isomorphism from $C^{*}\left(N_{1}\right)$ noto $C^{*}\left(N_{2}\right)$, then $\sigma(T)=\sigma(\alpha(T))$ for any $T$ in $C^{*}\left(N_{1}\right)$. To prove the converse, observe that, for a normal operator $N, C^{*}(N)$ is isometrically *-isomorphic to $C\left(\sigma(N)\right.$ ) (the $C^{*}$-algebra of all continuous functions on $\sigma(N))$ under the mapping $N \leftrightarrow \psi$, where $\psi(z)=z$ for all $z$ in $\sigma(N)$. The assertion follows from this observation immediately.

The following proposition follows easily from [3, Theorem 3].

Proposition 2.2. If $T_{1}$ and $T_{2}$ are isometries, then they are algebraically equivalent if and only if either they are both unitary with equal spectra or they are both nonunitary.

Proposition 2.3. Let $p$ be a polynomial in two noncommuting variables and $A_{j}>0, j=1,2$. If $\sigma\left(A_{1}\right)=\sigma\left(A_{2}\right)$, then $\left\|p\left(S \otimes A_{1},\left(S \otimes A_{1}\right)^{*}\right)\right\|=\| p\left(S \otimes A_{2},(S \otimes\right.$ $\left.\left.A_{2}\right)^{*}\right) \|$. 
Proof. Assume that $A_{2}$ is diagonal with eigenvalues $\left\{a_{n}\right\}$. The general case follows from it immediately. In this case, $S \otimes A_{2} \cong \sum_{n} \oplus a_{n} S$. A straightforward computation shows that

$$
p\left(S \otimes A_{2},\left(S \otimes A_{2}\right)^{*}\right) \cong \sum_{n} \oplus p\left(a_{n} S, a_{n} S^{*}\right),
$$

and hence

$$
\left\|p\left(S \otimes A_{2},\left(S \otimes A_{2}\right)^{*}\right)\right\|=\sup _{n}\left\|p\left(a_{n} S, a_{n} S^{*}\right)\right\| .
$$

For $\epsilon>0$, choose $\delta>0$ such that

$$
\left\|p\left(X, X^{*}\right)-p\left(Y, Y^{*}\right)\right\|<\epsilon \text { whenever }\|X\|,\|Y\| \leq\left\|A_{1}\right\| \text { and }\|X-Y\| \leq \delta .
$$

Assune that $A_{1}$ acts on Hilbert space $H$. Let $E_{A_{1}}$ be the spectral measure for $A_{1}$,

$$
B_{n}=A_{1} \mid E_{A_{1}}\left(\left(a_{n}-\delta, a_{n}+\delta\right)\right) H \text { and } D_{n}=a_{n} E_{A_{1}}\left(\left(a_{n}-\delta, a_{n}+\delta\right)\right) .
$$

Since

$$
\left\|S \otimes B_{n}-S \otimes D_{n}\right\|=\left\|B_{n}-D_{n}\right\| \leq \delta,
$$

it follows from (2.3.1) that

$$
\begin{aligned}
\left\|p\left(S \otimes A_{1},\left(S \otimes A_{1}\right)^{*}\right)\right\| & \geq\left\|p\left(S \otimes B_{n},\left(S \otimes B_{n}\right)^{*}\right)\right\| \\
& \geq\left\|p\left(S \otimes D_{n},\left(S \otimes D_{n}\right)^{*}\right)\right\|-\epsilon \\
& =\left\|p\left(a_{n} S, a_{n} S^{*}\right)\right\|-\epsilon
\end{aligned}
$$

for each $n$, and hence

$$
\begin{aligned}
\left\|p\left(S \otimes A_{1},\left(S \otimes A_{1}\right)^{*}\right)\right\| & \geq \sup _{n}\left\|p\left(a_{n} S, a_{n} S^{*}\right)\right\|-\epsilon \\
& =\left\|p\left(S \otimes A_{2},\left(S \otimes A_{2}\right)^{*}\right)\right\|-\epsilon .
\end{aligned}
$$

On the other hand, there exist $\left\{b_{j}\right\}_{j=1}^{m}$ with $0<b_{j}-b_{j-1} \leq \delta, b_{0}=0$ and $\sigma\left(A_{1}\right) \subset\left[0, b_{m}\right]$. Let

$$
A_{1 j}=A_{1} \mid E_{A_{1}}\left(\left[b_{j-1}, b_{j}\right)\right) H, \quad 1 \leq j<m
$$

and

$$
A_{1 m}=A_{1} \mid E_{A_{1}}\left(\left[b_{m-1}, b_{m}\right]\right) H .
$$

Then

$$
A_{1}=\sum_{j=1}^{m} \oplus A_{1 j}
$$

For each $j$, choose $N_{j}$ such that

$$
\left\|A_{1 j}-a_{n_{j}}\right\|<\delta
$$


Again, from (2.3.1), we have

$$
\left\|p\left(S \otimes A_{2},\left(S \otimes A_{2}\right)^{*}\right)\right\| \geq\left\|p\left(a_{n_{j}} S, a_{n_{j}} S^{*}\right)\right\| \geq\left\|p\left(S \otimes A_{1 j},\left(S \otimes A_{1 j}\right)^{*}\right)\right\|-\epsilon .
$$

Therefore,

$$
\left\|p\left(S \otimes A_{2},\left(S \otimes A_{2}\right)^{*}\right)\right\| \geq\left\|p\left(S \otimes A_{1},\left(S \otimes A_{1}\right)^{*}\right)\right\|-\epsilon .
$$

Since $\epsilon$ is arbitrary, we conclude, by (2.3.2) and (2.3.3), that

$$
\left\|p\left(S \otimes A_{2},\left(S \otimes A_{2}\right)^{*}\right)\right\|=\left\|p\left(S \otimes A_{1},\left(S \otimes A_{1}\right)^{*}\right)\right\|
$$

This completes the proof.

Lemma 2.4. If $p$ is a polynomial in two noncommuting variables and $A>0$; then $\left\|p\left(S \otimes A,(S \otimes A)^{*}\right)\right\| \geq \sup \{|p(\lambda, \bar{\lambda})|:|\lambda| \in \sigma(A)\}$.

Proof. Let $B$ be a diagonal operator with eigenvalues $\left\{a_{n}\right\}$ satisfying $\overline{\left\{a_{n}\right\}}=\sigma(A)$. Since $C^{*}(S) / \mathcal{K}(H)$ is an abelian $C^{*}$-algebra generated by $\pi(S)$, it is $*$-isomorphic to $C(\sigma(\pi(S)))=C(\partial D)([3$, Theorem 2]). We have

$$
\begin{aligned}
\left\|p\left(a_{n} S, a_{n} S^{*}\right)\right\| & \geq\left\|p\left(\pi\left(a_{n} S\right), \pi\left(a_{n} S^{*}\right)\right)\right\| \\
& =p\left(a_{n} \pi(S), a_{n} \pi\left(S^{*}\right)\right) \| \\
& =\sup \left\{\left|p\left(a_{n} \lambda, a_{n} \bar{\lambda}\right)\right|: \lambda \in \sigma(\pi(S))=\partial D\right\} \\
& =\sup \left\{\mid p\left(\lambda, \bar{\lambda}|:| \lambda \mid=a_{n}\right\} .\right.
\end{aligned}
$$

Proposition 2.3 leads to that

$$
\begin{aligned}
\left\|p\left(S \otimes A,(S \otimes A)^{*}\right)\right\| & =\left\|p\left(S \otimes B,(S \otimes B)^{*}\right)\right\| \\
& =\sup _{n}\left\|p\left(a_{n} S, a_{n} S^{*}\right)\right\| \\
& \geq \sup \left\{|p(\lambda, \bar{\lambda})|:|\lambda| \in\left\{a_{n}\right\}\right\} \\
& =\sup \{|p(\lambda, \bar{\lambda})|:|\lambda| \in \sigma(A)\}
\end{aligned}
$$

completing the proof.

The next theorem on algebraic equivalence for quasinormal operators generalizes Propositions 2.1 and 2.2.

Theorem 2.5. Let $T_{j}=N_{j} \oplus\left(S \otimes A_{j}\right)$ be quasinormal, where $N_{j}$ is normal and $A_{j}>0, j=1,2$. Then $T_{1}$ is algebraically equivalent to $T_{2}$ if and only if $\sigma\left(A_{1}\right)=\sigma\left(A_{2}\right)$ and $\sigma\left(N_{1}\right) \backslash \sigma_{a p}\left(S \otimes A_{1}\right)=\sigma\left(N_{2}\right) \backslash \sigma_{a p}\left(S \otimes A_{2}\right)$.

Proof. Let $\alpha$ be a *-isomorphism from $C^{*}\left(T_{1}\right)$ noto $C^{*}\left(T_{2}\right)$ such that $\alpha\left(T_{1}\right)=T_{2}$. We have $\alpha\left(T_{1}^{*} T_{1}-T_{1} T_{1}^{*}\right)=T_{2}^{*} T_{2}-T_{2} T_{2}^{*}$, that is $\alpha\left(A_{1}^{2} \oplus 0\right)=A_{2}^{2} \oplus 0$. Consequently, $\sigma\left(A_{1}^{2} \oplus 0\right)=\sigma\left(A_{2}^{2} \oplus 0\right)$. Therefore, $\sigma\left(A_{1}^{2}\right) \cup\{0\}=\sigma\left(A_{2}^{2}\right) \cup\{0\}$. Since 0 cannot be an isolated 
point in the spectrum of a positive definite operator, we conclude that $\sigma\left(A_{1}^{2}\right)=\sigma\left(A_{2}^{2}\right)$, and hence

$$
\sigma\left(A_{1}\right)=\sigma\left(A_{2}\right) \text {. }
$$

Next, observe that, for an operator $T, \lambda \notin \sigma_{a p}(T)$ is equivalent to that

$$
(T-\lambda)^{*}(T-\lambda) \geq \delta \text { for some } \delta>0 .
$$

This, together with order-preserving property of $\alpha$, shows that

$$
\sigma_{a p}\left(T_{1}\right)=\sigma_{a p}\left(T_{2}\right)
$$

Since

$$
\begin{aligned}
\sigma\left(N_{j}\right) \backslash \sigma_{a p}\left(S \otimes A_{j}\right) & =\left(\sigma\left(N_{j}\right) \cup \sigma_{a p}\left(S \otimes A_{j}\right)\right) \backslash \sigma_{a p}\left(S \otimes A_{j}\right) \\
& =\sigma_{a p}\left(T_{j}\right) \backslash \sigma_{a p}\left(S \otimes A_{j}\right), j=1,2,
\end{aligned}
$$

it follows from (2.5.1) and (2.5.2) that

$$
\sigma\left(N_{1}\right) \backslash \sigma_{a p}\left(S \otimes A_{1}\right)=\sigma\left(N_{2}\right) \backslash \sigma_{a p}\left(S \otimes A_{2}\right) .
$$

This proves the necessity condition:

To prove the sufficiency, it suffices to show that $\left\|p\left(T_{1}, T_{1}^{*}\right)\right\|=\left\|p\left(T_{2}, T_{2}^{*}\right)\right\|$ for any polynomial $p$. To do this, observe that

$$
\begin{aligned}
& \left\|p\left(T_{j}, T_{j}^{*}\right)\right\| \\
= & \left\|p\left(N_{j}, N_{j}^{*}\right) \oplus p\left(S \otimes A_{j},\left(S \otimes A_{j}\right)^{*}\right)\right\| \\
= & \max \left\{\left\|p\left(N_{j}, N_{j}^{*}\right)\right\|,\left\|p\left(S \otimes A_{j},\left(S \otimes A_{j}\right)^{*}\right)\right\|\right\} \\
= & \max \left\{\sup \left\{|p(\lambda, \bar{\lambda})|: \lambda \in \sigma\left(N_{j}\right)\right\},\left\|p\left(S \otimes A_{j},\left(S \otimes A_{j}\right)^{*}\right)\right\|\right\} \\
= & \max \left\{\sup \left\{\mid p(\lambda, \bar{\lambda}): \lambda \in \sigma\left(N_{j}\right) \backslash \sigma_{a p}\left(S \otimes A_{j}\right)\right\},\right. \\
& \left.\sup \left\{|p(\lambda, \bar{\lambda})|: \lambda \in \sigma\left(N_{j}\right) \cap \sigma_{a p}\left(S \otimes A_{j}\right)\right\},\left\|p\left(S \otimes A_{j},\left(S \otimes A_{j}\right)^{*}\right)\right\|\right\} \\
= & \max \left\{\sup \left\{|p(\lambda, \bar{\lambda})|: \lambda \in \sigma\left(N_{j}\right) \backslash \sigma_{a p}\left(S \otimes A_{j}\right)\right\},\left\|p\left(S \otimes A_{j},\left(S \otimes A_{j}\right)^{*}\right)\right\|\right\}
\end{aligned}
$$

by Lemma 2.4 and $\sigma_{a p}\left(S \otimes A_{j}\right)=\left\{Z:|z| \in \sigma\left(A_{j}\right)\right\}$ ([2, Lemma 2.2]). Our assumption and Proposition 2.3 imply that $\left\|p\left(T_{1}, T_{1}^{*}\right)\right\|=\left\|p\left(T_{2}, T_{2}^{*}\right)\right\|$. This completes the proof.

\section{References}

[1] A. Brown, "On a class of operators", Proc. Amer. Math. Soc., 4 (1953), 723-728.

[2] K. Y. Chen, Quasinormal Operators: Similarity, Quasismilarity and Compact Perturbation, Ph. D. dissertation, National Chiao Tung University, 1991.

[3] L. A. Coburn, "The $C^{*}$-algebra generated by an isometry", Bull. Amer. Math. Soc., 73 (1967), 722-726.

Departement of Mathematics, Tamkang University, Tamsui, Taiwan 25137, Republic of China. 